\title{
Design of IIR Digital Highpass Butterworth Filter using Analog to Digital Mapping Technique
}

\author{
Subhadeep Chakraborty \\ Department of ECE \\ Calcutta Institute of \\ Technology \\ Uluberia, Howrah, West \\ Bengal, India, PIN-711316
}

\author{
Krishna Kumar Jha \\ Department of MCA \\ Calcutta Institute of \\ Technology \\ Uluberia, Howrah, West \\ Bengal, India, PIN-711316
}

\author{
Abhirup Patra \\ Department of ECE \\ Calcutta Institute of \\ Technology \\ Uluberia, Howrah, West \\ Bengal, India, PIN-711316
}

\begin{abstract}
Infinite Impulse Response (IIR) filter is of recursive type filter. The present output sample of an IIR filter depends on the present input samples, past input samples and past output samples. There are a number of techniques available to determine the digital IIR Filters. This paper is based on the computer based approach to design the digital IIR filter along with the calculation of filter coefficients using the analog to digital mapping technique. The program based on the proposed algorithm is simulated in Matlab and found the result is satisfying.
\end{abstract}

Keywords: IIR filter, Digital filters, Butterworth filter, High pass filter, coefficient, analog to digital mapping

\section{INTRODUCTION}

Filters play a very important role in signal processing. In this paper the IIR digital filter is discussed which is very essential in Digital Signal Processing (DSP).In DSP, there are two type of systems. The first type of system performs signal filtering in time domain. They are known as Digital filters [1][9]. Another type of system provide signal representation in frequency domain. They are known to as Spectrum analyzer [1][3]. The term IIR comes from infinite impulse response meaning that the impulse response of filter is of infinite duration whereas the impulse response of a FIR (Finite Impulse Response) filter is of finite duration.

IIR filter processes certain properties such as width of the passband, width of the stop-band, maximum allowable ripple at pass-band and maximum allowable ripple at stopband[1][2][11]. A preferred design of IIR filter can be done with help of those properties [4]. There are various process to design IIR Digital filter. Basically IIR digital filter is designed from an analog filter. Then using analog to digital mapping technique or frequency transformation an IIR Digital filter can be designed suitably[2][4].

An analog filter generally constructed by resistors, capacitors and op amps to produce the required filtering effect. Those filter circuits can be widely used in reduction of noise, video signal enhancement, graphic equalizers in hi-fi systems, and many other areas. Those analog filters are actually designed as per specific requirement for producing satisfying filtering output. Digital filters perform many filtering works by replacing the analog filters. The digital filters have many features for which we can replace the analog filters and use the digital filters and the features are high accuracy and reliability, small physical size and reduced sensitivity to component tolerances or drift[5][13]. Analog filter can be designed by either active element or passive element. When the design of an active filter is completed, the cutoff frequency can be calculated. When the cutoff frequency is obtained, the pass-band or stop-band allowable frequency can easily be obtained. Now the IIR digital filter can be implemented directly, such as for example if a analog Low Pass Filter is designed and the designer wants to design a High Pass Filter, the designer can apply mapping technique and frequency transformation to transform from Low Pass Filter to High Pass Filter or the designer can design from analog High Pass Filter to High Pass Filter using frequency transformation technique.[2]

There are two transformation techniques available that are widely used are- Impulse invariant method and bilinear transformation [2][4].

\section{DIGITAL IIR FILTER DESIGN}

An IIR filter, as discussed in the introductory part, can be designed from active or passive element. When a voltage source is applied across the input terminal, the filter becomes an active filter. Now there are many type of IIR filters such as Butterworth filter, Chebyshev filter, Elliptic filter etc. A Butterworth filter designed by Opamp is shown in fig.1[5]

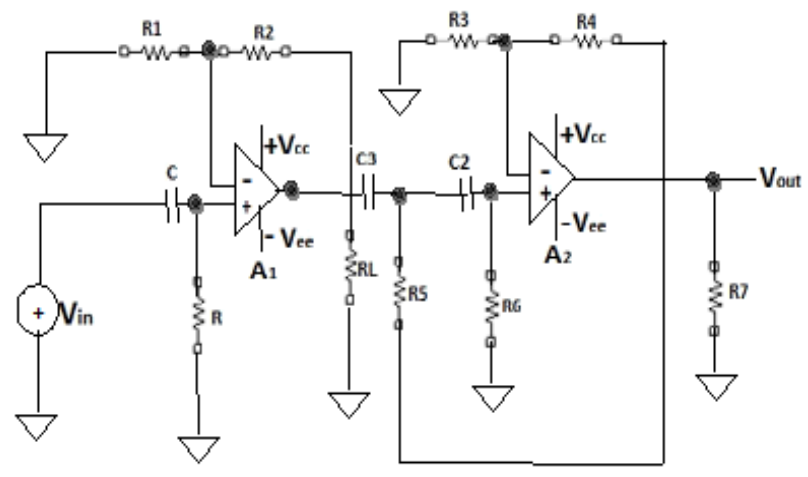

Fig.1 $3^{\text {rd }}$ Order IIR Highpass Butterworth Filter

In signal processing, the order of the FIR filter is always higher than that of the IIR filter when we basically view the same magnitude response. So in that case, the group delay of FIR filter is large enough compared to IIR filter. So, in order to process a signal processing with high-speed and with highprecision, it is very important to design the IIR filters [12].

Now from the Fig.1 [5], a digital IIR Filter can be designed. The left hand section is a $1^{\text {st }}$ Order section and the right hand section is a $2^{\text {nd }}$ order section. This is because the general available designs for filters are the $1^{\text {st }}$ Order filter and the $2^{\text {nd }}$ Order filter. Therefore by means of cascading those two a $3^{\text {rd }}$ Order filter can be designed [5]. 
A digital filter means its transfer function must be in the $\mathrm{z}$ plane, i.e. $\mathrm{H}(\mathrm{z})$. The impulse response $\mathrm{h}(\mathrm{n})$ for a realizable filter is,

$$
\mathrm{h}(\mathrm{n})=0 \quad \text { for } \mathrm{n} \leq 0
$$

A stable filter must satisfy the condition,

$$
\sum_{n=0}^{\infty}|h(n)|<\infty
$$

Now the generalized transfer function [1][2][10] of an IIR Digital filter is,

$$
H(z)=\frac{\sum_{n=0}^{M} b(n) z^{-n}}{1+\sum_{n=1}^{N} a(n) z^{-n}}
$$

$=\frac{B(z)}{A(z)}=\frac{b(0)+b(1) z^{-1}+b(2) z^{-2}+\ldots \ldots .+b(M) z^{-M}}{1+a(1) z^{-1}+a(2) z^{-2}+\ldots \ldots \ldots \ldots .+a(N) z^{-N}}$

Where,

$b(n)=$ Numerator coefficient of the filter $a(n)=$ Denominator coefficient of the filter

IIR filters have many advantages as follows[1]:-

It requires less number of arithmetic operations.

There are shorter time delays in these filters.

IIR Filters have similarities with analog filters.

These filters depend not only upon the input but also upon previous output values.

They are more susceptible to noises.

A suitable digital filter can design by calculating the numerator coefficient and denominator coefficient. There are various techniques available for the design and calculation of those coefficients. The algorithm proposed for this purpose is smartly eligible to determine the filter coefficients and hence helps to design the IIR Digital filter with correct specification provided to it. This algorithm also provide with the transfer function in the digital domain. The flowchart of the proposed algorithm is shown in fig. 2

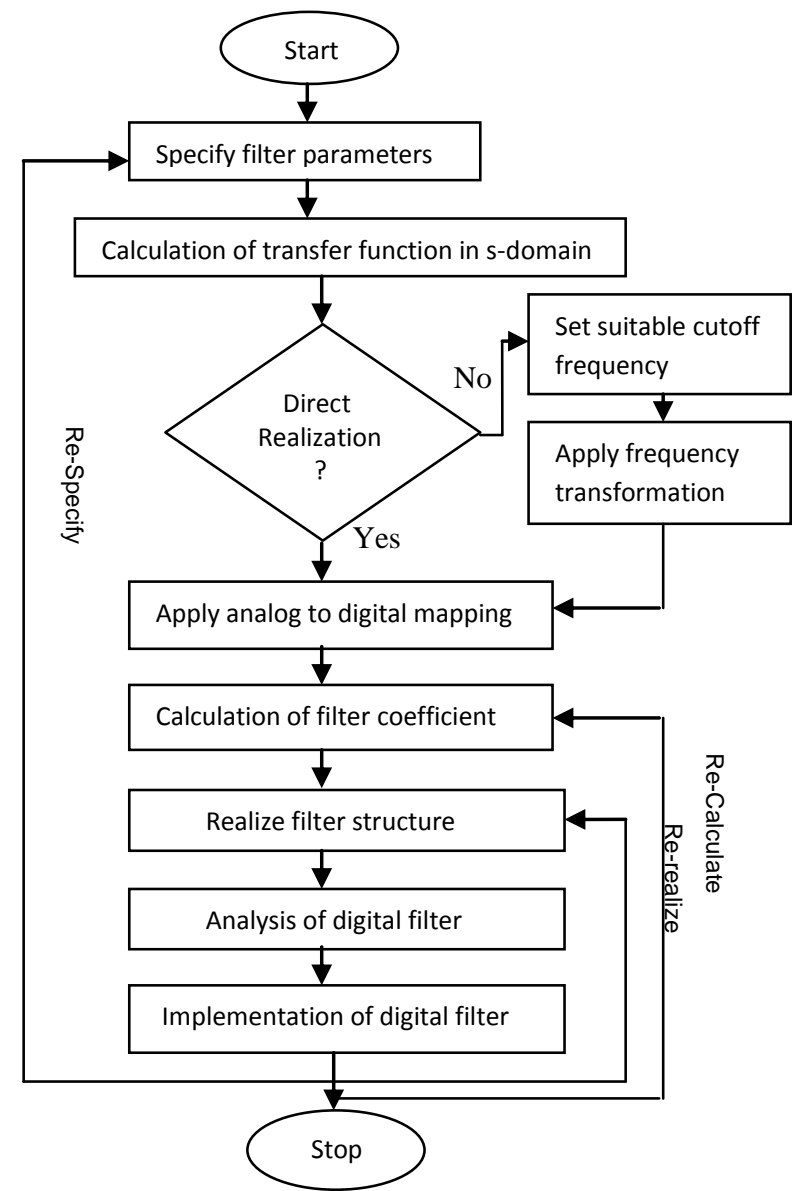

Fig.2 Proposed algorithm

Now, with help of this algorithm, the designer can specify the necessary parameters for a filter directly or the transformation from Low pass filter to High pass filter or Low pass filter to Low pass filter or High pass filter to Low pass filter or High pass to High pass filter are also allowed.

In the proposed algorithm, the direct and indirect realization of a digital filter can be performed. Let, the parameters of an analog filter is specified. Then after calculation the transfer function of the analog filter i.e. in s-domain is obtained. Then it's the designer choice whether he is interested in direct realization or in indirect realization. If the realization is the indirect one, the suitable cutoff frequency must be specified and on that the frequency transformation will be done. For direct realization, the analog to digital mapping will be performed on the calculated transfer function in s-domain so that it produces the final transfer function in z-domain which indicates the transfer function of a digital filter. Then by calculation, the filter coefficient can be determined .The stability of the digital filter is determined by the pole-zero plot. So by applying the algorithm, the digital stable filter can be determined. 


\section{ANALOG TO DIGITAL MAPPING}

Analog to Digital mapping means, simply, transformation of the transfer function of a specified circuit from the s-domain to $\mathrm{z}$-domain. If a filter i.e. its transfer function is designed in sdomain, it is called the analog filter. After mapping, when the transfer function is finally designed in $\mathrm{z}$-domain, this is called a digital filter.

Now to perform mapping, let we consider the impulse response of the filter in time domain is $h(t)$. So the transfer function corresponding to $h(t)$ can be obtained by the Laplace transform[6],i.e.

$$
H(s)=L\{h(t)\}=\int_{0}^{\infty} h(t) \cdot e^{-s t} d t
$$

Where,

$$
\begin{aligned}
\mathrm{s} & =\text { complex variable } \\
& =\sigma+\mathrm{j} \omega
\end{aligned}
$$

Here $h(t)$ is continuous. To obtain the discrete format of $h(t)$ i.e. $\mathrm{h}(\mathrm{n})$, substitute

$$
\mathrm{t}=\mathrm{nT}
$$

where,

$\mathrm{T}=$ sampling time

So, by substituting $t=n T$, we get $h(n T)$ from $h(t)$. If the sampling time $\mathrm{T}=1 \mathrm{sec}$, then we obtain the discrete form of $\mathrm{h}(\mathrm{t})$ as $h(n)$. Now from $h(n)$ we can easily design $H(z)$, i.e.,

$$
H(z)=Z\{h(n)\}=\sum_{n=-\infty}^{\infty} h(n) z^{-n}
$$

So, in this way, the transfer function of the digital filter can be obtained and moreover the stable poles of s-plane are mapped inside the unit circle of the z-plane[2]. Fig.3 shows the concept of mapping from s-plane to z-plane and vice versa.

The Butterworth High pass filter response increases logarithmically with increase in frequency and provides with

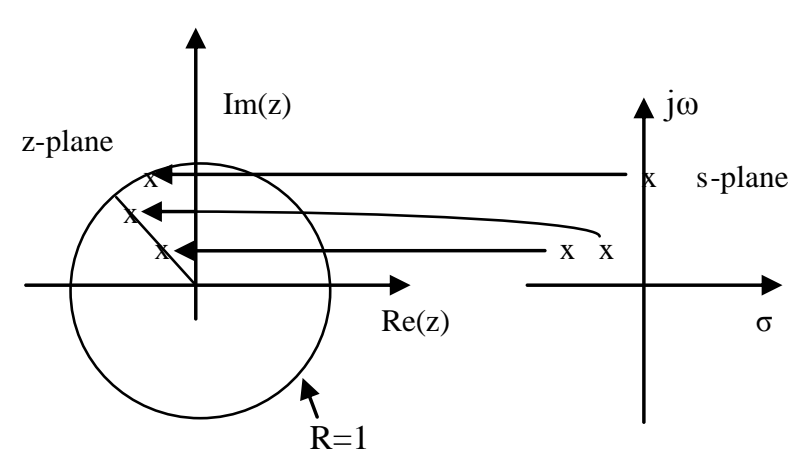

Fig.3 Mapping of poles monotonic amplitude frequency response at $0 \mathrm{db}$. The magnitude curve can be obtained from the software program by providing the coefficients to it and the coefficient is obtained by the mapping technique.

Basically the practical interest goes to the point that to determine the filter coefficients properly that in case truly help the designer to construct a filter of interest. By using the proposed algorithm, the filter coefficient can be determined.

\section{SIMULATION RESULT DISCUSSION}

The program for the design of IIR Butterworth High-pass filter is simulated in MATLAB7 by choosing the proper specifications such as Pass-band frequency, Stop-band frequency, Maximum allowable Pass-band and Stop-band ripples, so that the designed High-pass filter will be perfect. The output graphs are shown from Fig.4 to Fig.11.

Table 1 gives the results of the coefficients of High-pass Butterworth filter for order $=3$ and order $=5$.

Table.1 Result for coefficients

\begin{tabular}{|l|c|l|l|}
\hline \multirow{2}{*}{ Filter name } & $\begin{array}{c}\text { Filter } \\
\text { order }\end{array}$ & \multicolumn{1}{|c|}{$\begin{array}{l}\text { Numerator } \\
\text { coefficient }\end{array}$} & $\begin{array}{l}\text { Denominator } \\
\text { coefficient }\end{array}$ \\
\hline & & $-0.1247,-0.4272$, & $\begin{array}{l}-0.3616, \\
0.3602, \\
\text { Butterworth }\end{array}$ \\
$\begin{array}{l}\text { Highpass } \\
\text { Filter }\end{array}$ & & -0.4776 & -0.2123 \\
\cline { 2 - 4 } & & $\begin{array}{l}-0.5807,-0.424, \\
-1.548,-2.826,\end{array}$ & $-1.471,1.912$, \\
& 5 & -2.064 & $-0.8353,1.355$, \\
& & & \\
\hline
\end{tabular}


$\underline{\text { IIR Butterworth High-pass Filter(Order }=3 \text { ) }}$

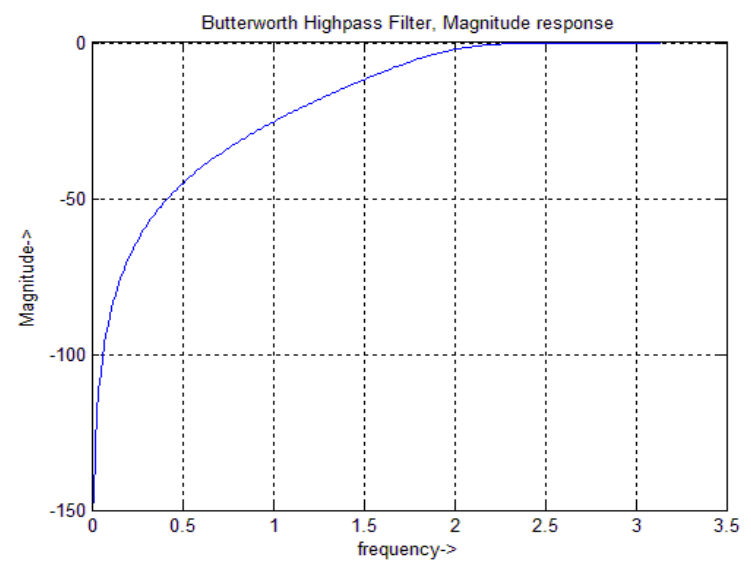

Fig.4 Magnitude response(Order=3)

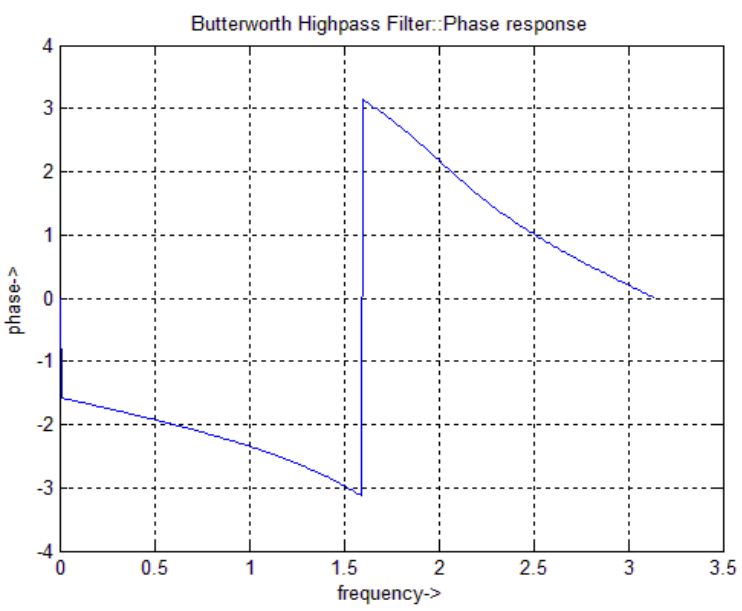

Fig.6 Phase response(Order=3)

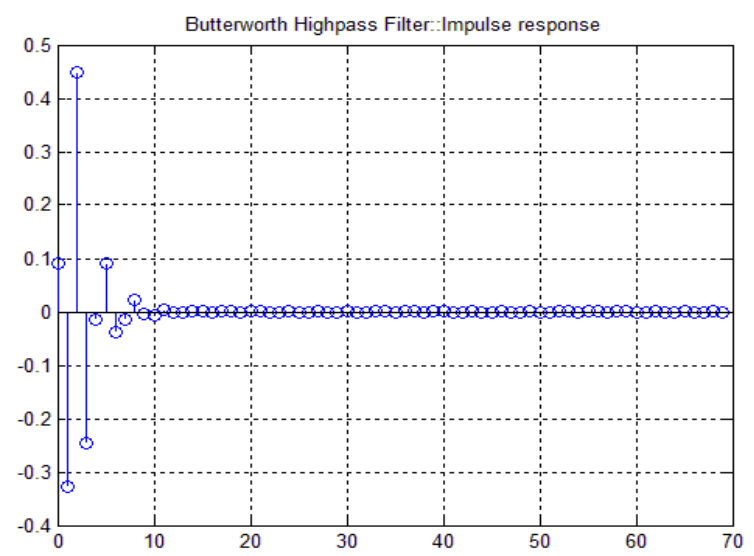

Fig.8 Impulse response(Order=3) $\underline{\text { IIR Butterworth High-pass Filter(Order = 5) }}$

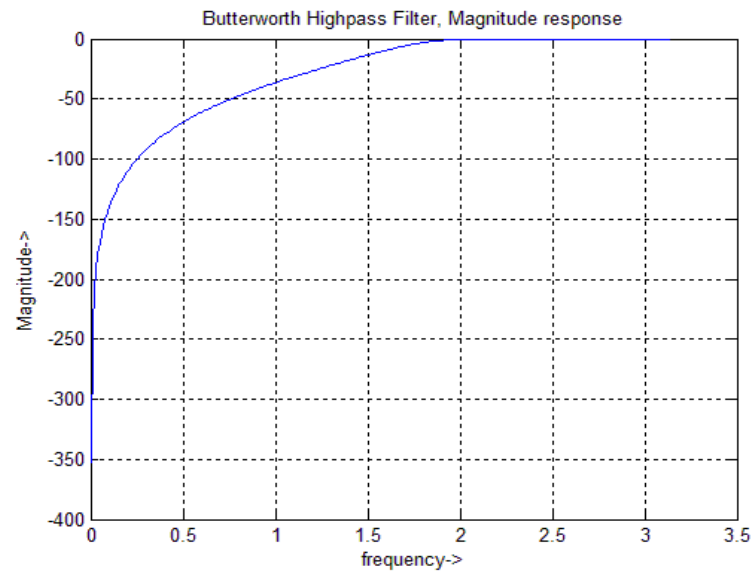

Fig.5 Magnitude response(Order=5)

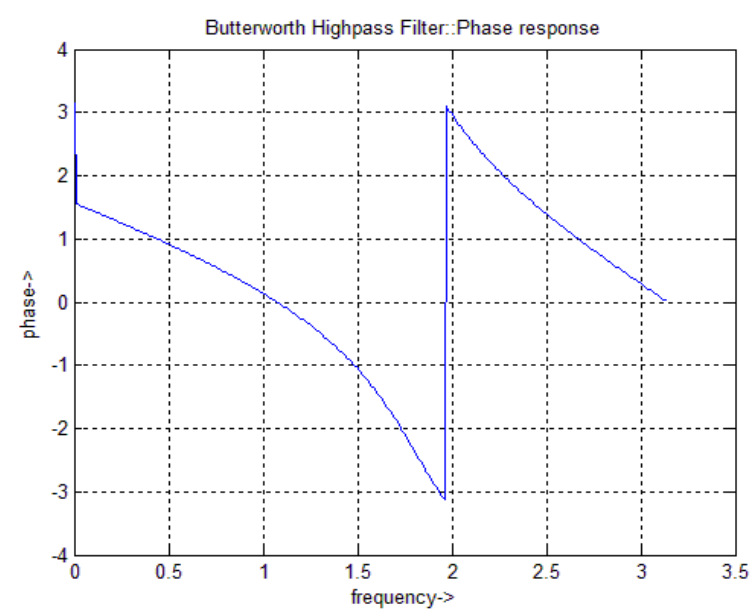

Fig.7 Phase response(Order=5)

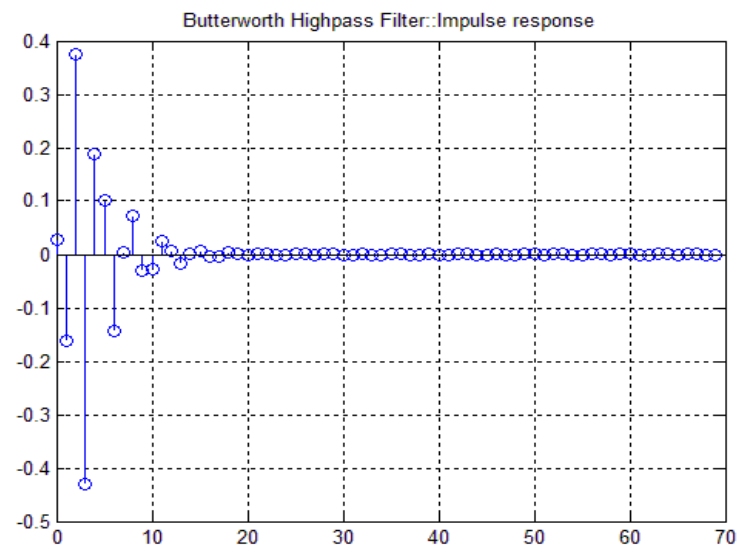

Fig.9 Impulse response(Order=5) 


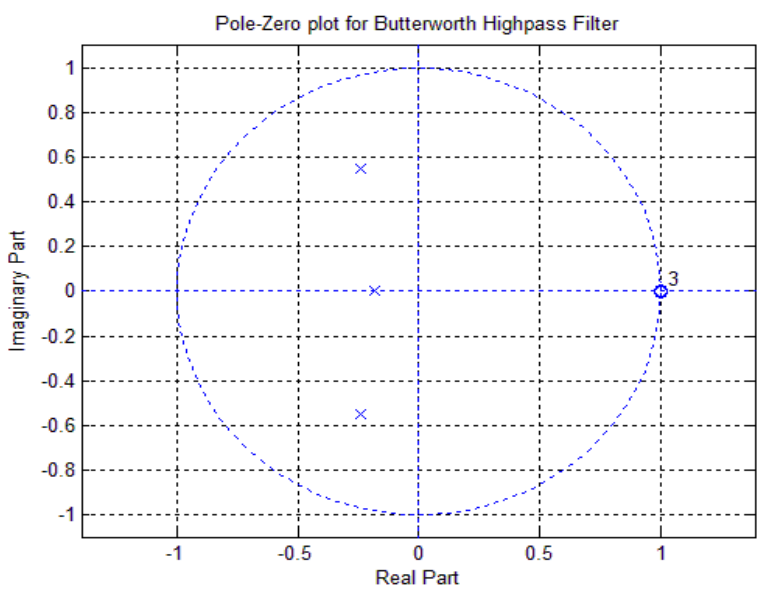

Fig.10 Pole-Zero plot(Order=3)

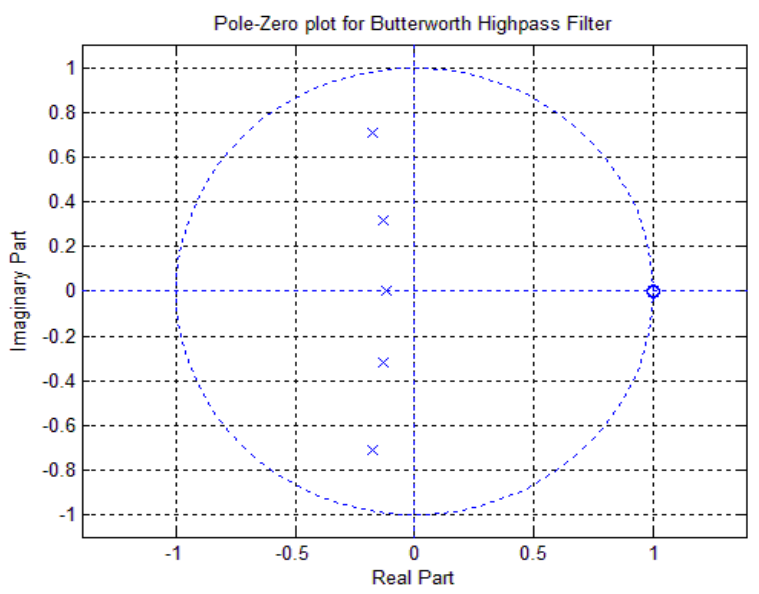

Fig.11 Pole-Zero plot(Order=5)

\section{CONCLUSION}

In this paper, the calculation and result for the coefficients of IIR Butterworth High-pass filter using the analog to digital mapping is shown. Those coefficients, that are determined by the proposed algorithm, are truly necessary for designing the filter. Now, if we look on the pole-zero plot of the filter, we can see that the filters are stable. So, we can design an analog filter to a stable digital filter by applying analog to digital mapping technique and with help of suitable proposed algorithm by which a stable filter as well as the optimum values of its coefficients is obtained and the output figures are presented in above figures, generated by Matlab 7.

\section{REFERENCES}

[1]. Ranjit Singh and Sandeep K. Arya, "Determining Optimum coefficients of IIR Digital Filter using Analog to Digital Mapping," International Journal of Advancements in Computer Science and Information Technology, Vol. 01,No. 01, September 2011 pp.19-23.

[2]. P. Ramesh Babu,'Digital Signal Processing", Fourth edition, Scitech Publication(India) Pvt. Ltd, Chennai,2008.

[3]. R.S. Chauhan and Sandeep K. Arya,'Design of IIR digital filter using analog to digital mapping", Journal of Neural Computing Systems, Vol. 03,No. 01,2010,pp. 51-55.

[4]. Yaduvir Singh, Sweta tripathi and Manoj Pandey, " Analysis of Digital IIR filter with LabVIEW", International Journal of Computer Applications, Vol. 10, No. 06, 2010, pp.23-30.

[5]. Ramakant A. Gayakwad, "Opamp and Linear Integrated Circuit”, Fourth Edition, PHI Learning Private Limited, New Delhi, 2010.

[6] A. Sudhakar and Shyammohan S. Palli, "Circuits and Network", Fourth Edition, TataMcGraw Hill Education Private Limited, New Delhi, 2011.

[7]. Samarjeet Singh and Uma Sharma, "Matlab based Digital IIR filter design", International Journal of Electronics and Computer Science Engineering, Vol. 01, No. 01,ISSN 2277-1956,pp.74-83

[8] Proakis, J. G. and Manolakis, D. G. 2007. Digital Signal Processing: Principles, Algorithms, and Applications. Pearson Education Ltd.

[9] Ranjit Singh Chauhan and Sandeep Kumar Arya, "Determine Optimal Coefficients of IIR Digital Filters using Simulated Annealing", International Journal of Computer Applications (0975 - 8887) Volume 43No.10, April 201236

[10] Amar Palacherla, Microchip Technology Inc, "Implementing IIR Digital Filters", Microchip Technology Inc.

[11] Ranjit Kaur, Manjeet Singh Patterh and J.S. Dhillon, "Design of Optimal L1 Stable IIR Digital Filter using Hybrid Optimization Algorithm", International Journal of Computer Applications (0975 - 8887) Volume 38 No.2, January 2012

[12] Yasunori Sugita and Toshinori Yoshikawa, "Design of Stable IIR Digital Filters with Specified Group Delay Errors", International Journal of Information and Communication Engineering 6:1 2010

[13] Gurleen Kaur and Ranjit Kaur, "Design of Recursive digital filters using Multiobjective Genetic algorithm", Gurleen Kaur et al. / International Journal of Engineering Science and Technology (IJEST), ISSN : 0975-5462 Vol. 3 No. 7 July 2011

\section{AUTHOR'S PROFILE}

Subhadeep Chakraborty, born in 1986, is Assistant Professor in Calcutta Institute of Technology. He received the B.Tech degree from Saroj Mohan Institute of Technology, WBUT,India and M.Tech degree from Kalyani Govt. Engineering College, WBUT, India in Electronics and Communication Engineering in 2008 and 2010.The author has been teaching in Calcutta Institute of Technology for 2 years. His primary research interest includes Digital Signal Processing, Embedded System and Microprocessor.

Krishna Kumar Jha received the B. Sc. degree from University of Calcutta, India, 2001 and the Master in Computer Application [MCA] from Sikkim Manipal University of Health, Medical \& Technological Sciences, 
Gangtok, India, 2008 and Master of Technology - Computer Science and Application from University of Calcutta, India, 2011. Currently he is Asst Prof. in the Dept of MCA at Calcutta Institute Of Technology, Uluberia, India. The author has been teaching for the last 7 years in the field of computer science. His primary research area includes ns2, WMN, cloud computing, virtualization of Network.
Abhirup Patra is pursuing his B.Tech degree from Calcutta Institute of Technology and this is his final year. His basic interest includes Digital Signal Processing, Control System. 